NBER WORKING PAPER SERIES

\title{
DYNAMIC TRADING WITH PREDICTABLE RETURNS AND TRANSACTION COSTS
}

\author{
Nicolae B. Garleanu \\ Lasse H. Pedersen \\ Working Paper 15205 \\ http://www.nber.org/papers/w15205
NATIONAL BUREAU OF ECONOMIC RESEARCH
1050 Massachusetts Avenue
Cambridge, MA 02138

August 2009

We are grateful for helpful comments from Kerry Back, Darrell Due, Pierre Collin-Dufresne, Andrea Frazzini, Esben Hedegaard, Hong Liu (discussant), Anthony Lynch, Ananth Madhavan (discussant), Andrei Shleifer, and Humbert Suarez, as well as from seminar participants at Stanford Graduate School of Business, University of California at Berkeley, Columbia University, NASDAQ OMX Economic Advisory Board Seminar, University of Tokyo, New York University, the University of Copenhagen, Rice University, University of Michigan Ross School, Yale University SOM, the Bank of Canada, and the Journal of Investment Management Conference. Lasse Pedersen is affiliated with AQR Capital Management, a global asset management firm that may apply some of the principles discussed in this research in some of its investment products. The views expressed herein are those of the author(s) and do not necessarily reflect the views of the National Bureau of Economic Research.

NBER working papers are circulated for discussion and comment purposes. They have not been peerreviewed or been subject to the review by the NBER Board of Directors that accompanies official NBER publications.

(C) 2009 by Nicolae B. Garleanu and Lasse H. Pedersen. All rights reserved. Short sections of text, not to exceed two paragraphs, may be quoted without explicit permission provided that full credit, including $\odot$ notice, is given to the source. 
Dynamic Trading with Predictable Returns and Transaction Costs

Nicolae B. Garleanu and Lasse H. Pedersen

NBER Working Paper No. 15205

August 2009

JEL No. G11,G12

\begin{abstract}
$\underline{\text { ABSTRACT }}$
We derive a closed-form optimal dynamic portfolio policy when trading is costly and security returns are predictable by signals with different mean-reversion speeds. The optimal strategy is characterized by two principles: 1) aim in front of the target and 2) trade partially towards the current aim. Specifically, the optimal updated portfolio is a linear combination of the existing portfolio and an "aim portfolio," which is a weighted average of the current Markowitz portfolio (the moving target) and the expected Markowitz portfolios on all future dates (where the target is moving). Intuitively, predictors with slower mean reversion (alpha decay) get more weight in the aim portfolio. We implement the optimal strategy for commodity futures and find superior net returns relative to more naive benchmarks.
\end{abstract}

Nicolae B. Garleanu

Haas School of Business

F628

University of California, Berkeley

Berkeley, CA 94720

and NBER

garleanu@haas.berkeley.edu

Lasse H. Pedersen

Copenhagen Business School

Solbjerg Plads 3, A5

DK-2000 Frederiksberg

DENMARK

and NYU

and also NBER

lpederse@stern.nyu.edu 
Active investors and asset managers — such as hedge funds, mutual funds, and proprietary traders - try to predict security returns and trade to profit from their predictions. Such dynamic trading often entails significant turnover and transaction costs. Hence, any active investor must constantly weigh the expected benefit of trading against its costs and risks. An investor often uses different return predictors, e.g., value and momentum predictors, and these have different prediction strengths and mean-reversion speeds, or, said differently, different "alphas" and "alpha decays." The alpha decay is important because it determines how long the investor can enjoy high expected returns and, therefore, affects the trade-off between returns and transactions costs. For instance, while a momentum signal may predict that the IBM stock return will be high over the next month, a value signal might predict that Cisco will perform well over the next year.

This paper addresses how the optimal trading strategy depends on securities' current expected returns, the evolution of expected returns in the future, their risks and correlations, and their transaction costs. We present a closed-form solution for the optimal dynamic portfolio strategy, giving rise to two principles: 1) aim in front of the target and 2) trade partially towards the current aim.

To see the intuition for these portfolio principles, note that the investor would like to keep his portfolio close to the optimal portfolio in the absence of transaction costs, which we call the "Markowitz portfolio." The Markowitz portfolio is a moving target, since the return-predicting factors change over time. Due to transaction costs, it is obviously not optimal to trade all the way to the target all the time. Hence, transaction costs make it optimal to slow down trading and, interestingly, to modify the aim, thus not to trade directly towards the current Markowitz portfolio. Indeed, the optimal strategy is to trade towards an "aim portfolio," which is a weighted average of the current Markowitz portfolio (the moving target) and the expected Markowitz portfolios on all future dates (where the target is moving).

While new to finance, these portfolio principles have close analogues in other fields such as the guidance of missiles towards moving targets, shooting, and sports. For example, related 
dynamic programming principles are used to guide missiles to an enemy airplane in so-called "lead homing" systems. Similarly, hunters are reminded to "lead the duck" when aiming their weapon. ${ }^{1}$ The most famous example from the sports world is perhaps the following quote from the "great one":

"A great hockey player skates to where the puck is going to be, not where it is." - Wayne Gretzky

Another way to state our portfolio principle is that the best new portfolio is a combination of 1) the current portfolio (to reduce turnover), 2) the Markowitz portfolio (to partly get the best current risk-return trade-off), and 3) the expected optimal portfolio in the future (a dynamic effect).

Figure 1 illustrates this natural trading rule. The solid line illustrates the expected path [Figure 1] of the Markowitz portfolio, starting with large positions in both security 1 and security 2, and gradually converging towards its long-term mean (e.g., the market portfolio). The aim portfolio is a weighted-average of the current and future Markowitz portfolios so it lies in the "convex hull" of the solid line or, equivalently, between the current Markowitz portfolio and the expected aim portfolio next period. The optimal new position is achieved by trading partially towards this aim portfolio.

In this example, the curvature of the solid line means that the Markowitz position in security 1 decays more slowly as the predictor that currently "likes" security 1 is more persistent. Therefore, the aim portfolio has a larger position in security 1, and, consequently, the optimal trade buys more shares in security 1 than it would otherwise. We show that it is in fact a more general principle that predictors with slower mean reversion (alpha decay) get more weight in the aim portfolio. An investor facing transaction costs should trade more aggressively on persistent signals than on fast mean-reverting signals: the benefits from the former accrue over longer periods, and are therefore larger.

The key role played by each return predictor's mean reversion is an important implication of our model. It arises because transaction costs imply that the investor cannot easily change his portfolio and, therefore, must consider his optimal portfolio both now and in the future. 
In contrast, absent transaction costs, the investor can re-optimize at no cost and needs to consider only the current investment opportunities without regard to alpha decay.

Our specification of transaction costs is sufficiently rich to allow for both purely transitory and persistent costs. Persistent transaction costs means that trading leads to a market impact and this effect on prices persists for a while. Indeed, since we focus on marketimpact costs, it may be more realistic to consider such persistent effects, especially over short time periods. We show that, with persistent transaction costs, the optimal strategy remains to trade partially towards an aim portfolio and to aim in front of the target, though the precise trading strategy is different and more involved.

Finally, we illustrate our results empirically in the context of commodity futures markets. We use returns over the past five days, 12 months, and five years to predict returns. The five-day signal is quickly mean reverting (fast alpha decay), the 12-month signal mean reverts more slowly, whereas the five-year signal is the most persistent. We calculate the optimal dynamic trading strategy taking transaction costs into account and compare its performance to the optimal portfolio ignoring transaction costs and to a class of strategies that perform static (one-period) transaction-cost optimization. Our optimal portfolio performs the best net of transaction costs among all the strategies that we consider. Its net Sharpe ratio is about $20 \%$ better than that of the best strategy among all the static strategies. Our strategy's superior performance is achieved by trading at an optimal speed and by trading towards an aim portfolio that is optimally tilted towards the more persistent return predictors.

We also study the impulse-response of the security positions following a shock to return predictors. While the no-transaction-cost position immediately jumps up and mean reverts with the speed of the alpha decay, the optimal position increases more slowly to minimize trading costs and, depending on the alpha decay speed, may eventually become larger than the no-transaction-cost position, as the optimal position is reduced more slowly.

The paper is organized as follows. Section I describes how our paper contributes to the portfolio-selection literature that started with Markowitz (1952). We provide a closedform solution for a model with multiple correlated securities and multiple return predictors 
with different mean-reversion speeds. The closed-form solution illustrates several intuitive portfolio principles that are difficult to see in the models following Constantinides (1986), where the solution requires complex numerical techniques even with a single security and no return predictors (i.i.d. returns). Indeed, we uncover the role of alpha decay and the intuitive aim-in-front-of-the-target and trade-towards-the-aim principles, and our empirical analysis suggests that these principles are useful.

Section II lays out the model with temporary transaction costs and lays out the solution method. Section III shows the optimality of aiming in front of the target, and trading partially towards the aim. Section IV solves the model with persistent transaction costs. Section V provides a number of theoretical applications, while Section VI applies our framework empirically to trading commodity futures. Section VII concludes, and all proofs are in the appendix.

\section{Related Literature}

A large literature studies portfolio selection with return predictability in the absence of trading costs (see, e.g., Campbell and Viceira (2002) and references therein). Alpha decay plays no role in this literature, and nor does it play a role in the literature on optimal portfolio selection with trading costs but without return predictability following Constantinides (1986).

This latter literature models transaction costs as proportional bid-ask spreads and relies on numerical solutions. Constantinides (1986) considers a single risky asset in a partial equilibrium and studies trading-cost implications for the equity premium. ${ }^{2}$ Equilibrium models with trading costs include Amihud and Mendelson (1986), Vayanos (1998), Vayanos and Vila (1999), Lo, Mamaysky, and Wang (2004), and Gârleanu (2009), as well as Acharya and

Pedersen (2005), who also consider time-varying trading costs. Liu (2004) determines the optimal trading strategy for an investor with constant absolute risk aversion (CARA) and many independent securities with both fixed and proportional costs (without predictability). The assumptions of CARA and independence across securities imply that the optimal 
position for each security is independent of the positions in the other securities.

Our trade-partially-towards-the-aim strategy is qualitatively different from the optimal strategy with proportional or fixed transaction costs, which exhibits periods of no trading. Our strategy mimics a trader who is continuously "floating" limit orders close to the midquote - a strategy that is used in practice. The trading speed (the limit orders' "fill rate" in our analogy) depends on how large transaction costs the trader is willing to accept (i.e., on where the limit orders are placed).

In a third (and most related) strand of literature, using calibrated numerical solutions, trading costs are combined with incomplete markets by Heaton and Lucas (1996), and with predictability and time-varying investment-opportunity sets by Balduzzi and Lynch (1999), Lynch and Balduzzi (2000), Jang, Koo, Liu, and Loewenstein (2007), and Lynch and Tan (2011). Grinold (2006) derives the optimal steady-state position with quadratic trading costs and a single predictor of returns per security. Like Heaton and Lucas (1996) and Grinold (2006), we also rely on quadratic trading costs.

A fourth strand of literature derives the optimal trade execution, treating the asset and quantity to trade as given exogenously (see, e.g., Perold (1988), Bertsimas and Lo (1998), Almgren and Chriss (2000), Obizhaeva and Wang (2006), and Engle and Ferstenberg (2007)).

Finally, quadratic programming techniques are also used in macroeconomics and other fields, and, usually, the solution comes down to algebraic matrix Riccati equations (see, e.g., Ljungqvist and Sargent (2004) and references therein). We solve our model explicitly, including the Riccati equations.

\section{Model and Solution}

We consider an economy with $S$ securities traded at each time $t \in\{0,1,2, \ldots\}$. The securities' price changes between times $t$ and $t+1$ in excess of the risk-free return, $p_{t+1}-$ $\left(1+r^{f}\right) p_{t}$, are collected in an $S \times 1$ vector $r_{t+1}$ given by

$$
r_{t+1}=B f_{t}+u_{t+1} \text {. }
$$


Here, $f_{t}$ is a $K \times 1$ vector of factors that predict returns, ${ }^{3} B$ is an $S \times K$ matrix of factor loadings, and $u_{t+1}$ is the unpredictable zero-mean noise term with variance $\operatorname{var}_{t}\left(u_{t+1}\right)=\Sigma$.

The return-predicting factor $f_{t}$ is known to the investor already at time $t$ and it evolves according to

$$
\Delta f_{t+1}=-\Phi f_{t}+\varepsilon_{t+1},
$$

where $\Delta f_{t+1}=f_{t+1}-f_{t}$ is the change in the factors, $\Phi$ is a $K \times K$ matrix of meanreversion coefficients for the factors, and $\varepsilon_{t+1}$ is the shock affecting the predictors with variance $\operatorname{var}_{t}\left(\varepsilon_{t+1}\right)=\Omega$. We impose on $\Phi$ standard conditions sufficient to ensure that $f$ is stationary.

The interpretation of these assumptions is straightforward: the investor analyzes the securities and his analysis results in forecasts of excess returns. The most direct interpretation is that the investor regresses the return on security $s$ on the factors $f$ that could be past returns over various horizons, valuation ratios, and other return-predicting variables, and thus estimates each variable's ability to predict returns as given by $\beta^{\text {sk }}$ (collected in the matrix $B$ ). Alternatively, one can think of each factor as an analyst's overall assessment of the various securities (possibly based on a range of qualitative information) and $B$ as the strength of these assessments in predicting returns.

Trading is costly in this economy and the transaction cost $(T C)$ associated with trading $\Delta x_{t}=x_{t}-x_{t-1}$ shares is given by

$$
T C\left(\Delta x_{t}\right)=\frac{1}{2} \Delta x_{t}^{\top} \Lambda \Delta x_{t},
$$

where $\Lambda$ is a symmetric positive-definite matrix measuring the level of trading costs. ${ }^{4}$ Trading costs of this form can be thought of as follows. Trading $\Delta x_{t}$ shares moves the (average) price by $\frac{1}{2} \Lambda \Delta x_{t}$, and this results in a total trading cost of $\Delta x_{t}$ times the price move, which gives $T C$. Hence, $\Lambda$ (actually, $1 / 2 \Lambda$, for convenience) is a multi-dimensional version of Kyle's lambda, which can also be justified by inventory considerations (e.g., Grossman and Miller 
(1988) or Greenwood (2005) for the multi-asset case). While this transaction-cost specification is chosen partly for tractability, the empirical literature generally finds transaction costs to be convex (e.g., Engle, Ferstenberg, and Russell (2008), Lillo, Farmer, and Mantegna (2003)), with some researchers actually estimating quadratic trading costs (e.g., Breen, Hodrick, and Korajczyk (2002)).

Most of our results hold with this general transaction cost function, but some of the resulting expressions are simpler in the following special case.

Assumption A. Transaction costs are proportional to the amount of risk, $\Lambda=\lambda \Sigma$.

This assumption means that the transaction cost matrix $\Lambda$ is some scalar $\lambda>0$ times the variance-covariance matrix of returns, $\Sigma$, as is natural and, in fact, implied by the model of Gârleanu, Pedersen, and Poteshman (2009). To understand this, suppose that a dealer takes the other side of the trade $\Delta x_{t}$, holds this position for a period of time and "lays it off" at the end of the period. Then the dealer's risk is $\Delta x_{t}^{\top} \Sigma \Delta x_{t}$ and the trading cost is the dealer's compensation for risk, depending on the dealer's risk aversion reflected by $\lambda$.

The investor's objective is to choose the dynamic trading strategy $\left(x_{0}, x_{1}, \ldots\right)$ to maximize the present value of all future expected excess returns, penalized for risks and trading costs:

$$
\max _{x_{0}, x_{1}, \ldots} E_{0}\left[\sum_{t}(1-\rho)^{t+1}\left(x_{t}^{\top} r_{t+1}-\frac{\gamma}{2} x_{t}^{\top} \Sigma x_{t}\right)-\frac{(1-\rho)^{t}}{2} \Delta x_{t}^{\top} \Lambda \Delta x_{t}\right]
$$

where $\rho \in(0,1)$ is a discount rate, and $\gamma$ is the risk-aversion coefficient. ${ }^{5}$

We solve the model using dynamic programming. We start by introducing a value function $V\left(x_{t-1}, f_{t}\right)$ measuring the value of entering period $t$ with a portfolio of $x_{t-1}$ securities and observing return-predicting factors $f_{t}$. The value function solves the Bellman equation:

$V\left(x_{t-1}, f_{t}\right)=\max _{x_{t}}\left\{-\frac{1}{2} \Delta x_{t}^{\top} \Lambda \Delta x_{t}+(1-\rho)\left(x_{t}^{\top} E_{t}\left[r_{t+1}\right]-\frac{\gamma}{2} x_{t}^{\top} \Sigma x_{t}+E_{t}\left[V\left(x_{t}, f_{t+1}\right)\right]\right)\right\}$.

The model in its general form can be solved explicitly: 
Proposition 1 The model has a unique solution and the value function is given by

$$
V\left(x_{t}, f_{t+1}\right)=-\frac{1}{2} x_{t}^{\top} A_{x x} x_{t}+x_{t}^{\top} A_{x f} f_{t+1}+\frac{1}{2} f_{t+1}^{\top} A_{f f} f_{t+1}+A_{0} .
$$

The coefficient matrices $A_{x x}, A_{x f}$, and $A_{f f}$ are stated explicitly in (A.15), (A.18), and (A.22), and $A_{x x}$ is positive definite. ${ }^{6}$

\section{Results: Aim in Front of the Target}

We next explore the properties of the optimal portfolio policy, which turns out to be intuitive and relatively simple. The core idea is that the investor aims to achieve a certain position, but trades only partially towards this "aim portfolio" due to transaction costs. The aim portfolio itself combines the current optimal portfolio in the absence of transaction costs and the expected future such portfolios. The formal results are stated in the following propositions.

Proposition 2 (Trade Partially Towards the Aim) (i) The optimal portfolio is

$$
x_{t}=x_{t-1}+\Lambda^{-1} A_{x x}\left(\text { aim }_{t}-x_{t-1}\right) \text {, }
$$

which implies trading at a proportional rate given by the the matrix $\Lambda^{-1} A_{x x}$ towards the "aim portfolio,"

$$
\operatorname{aim}_{t}=A_{x x}^{-1} A_{x f} f_{t}
$$

(ii) Under Assumption A, the optimal trading rate is the scalar $a / \lambda<1$, where

$$
a=\frac{-(\gamma(1-\rho)+\lambda \rho)+\sqrt{(\gamma(1-\rho)+\lambda \rho)^{2}+4 \gamma \lambda(1-\rho)^{2}}}{2(1-\rho)} .
$$

The trading rate is decreasing in transaction costs $\lambda$ and increasing in risk aversion $\gamma$. 
This proposition provides a simple and appealing trading rule. The optimal portfolio is a weighted average of the existing portfolio $x_{t-1}$ and the aim portfolio:

$$
x_{t}=\left(1-\frac{a}{\lambda}\right) x_{t-1}+\frac{a}{\lambda} \operatorname{aim}_{t}
$$

The weight of the aim portfolio — which we also call the "trading rate" — determines how far the investor should rebalance towards the aim. Interestingly, the optimal portfolio always rebalances by fixed fraction towards the aim (i.e., the trading rate is independent of the current portfolio $x_{t-1}$ or past portfolios). The optimal trading rate is naturally greater if transaction costs are smaller. Said differently, high transaction costs imply that one must trade more slowly. Also, the trading rate is greater if risk aversion is larger, since a larger risk aversion makes the risk of deviating from the aim more painful. A larger absolute risk aversion can also be viewed as a smaller investor, for whom transaction costs play a smaller role, and who therefore trades closer to her aim.

Next, we want to understand the aim portfolio. The aim portfolio in our dynamic setting turns out to be closely related to the optimal portfolio in a static model without transaction costs $(\Lambda=0)$, which we call the Markowitz portfolio. In agreement with the classical findings of Markowitz (1952),

$$
\text { Markowitz }_{t}=(\gamma \Sigma)^{-1} B f_{t}
$$

As is well known, the Markowtiz portfolio is the tangency portfolio appropriately leveraged depending on the risk aversion $\gamma$.

Proposition 3 (Aim in Front of the Target) (i) The aim portfolio is the weighted average of the current Markowitz portfolio and the expected future aim portfolio. Under Assumption $A$, this can be written as follows, letting $z=\gamma /(\gamma+a)$ :

$$
\text { aim }_{t}=z \text { Markowitz }_{t}+(1-z) E_{t}\left(\text { aim }_{t+1}\right) .
$$

(ii) The aim portfolio can also be expressed as the weighted average of the current Markowitz 
portfolio and the expected Markowitz portfolios at all future times. Under Assumption A,

$$
\text { aim }_{t}=\sum_{\tau=t}^{\infty} z(1-z)^{\tau-t} E_{t}\left(\text { Markowitz }_{\tau}\right) .
$$

The weight $z$ of the current Markowitz portfolio decreases with the transaction costs $(\lambda)$ and increases in risk aversion $(\gamma)$.

We see that the aim portfolio is a weighted average of current and future expected Markowitz portfolios. While, without transaction costs, the investor would like to hold the Markowitz portfolio to earn the highest possible risk-adjusted return, with transaction costs the investor needs to economize on trading and thus trade partially towards the aim and, as a result, he needs to adjust his aim in front of the target. Proposition 3 shows that the optimal aim portfolio is an exponential average of current and future (expected) Markowitz portfolios, where the weight on the current (and near term) Markowitz portfolio is larger if transaction costs are smaller.

A graphical illustration of optimal trading rule. The optimal trading policy is [Figure 2] illustrated in detail in Figure 2. Panel A of Figure 2 shows how the optimal first trade is derived, Panel B shows the expected second trade, and Panel C shows the entire path of expected future trades. Let's first understand Panel A. The solid curve is the expected path of future Markowitz portfolios. Since expected returns mean revert, the expected Markowitz portfolio converges to its long-term mean, illustrated at the origin of the figure. In this example, asset 2 loads on a factor that decays the fastest, so the future Markowitz positions are expected to have relatively larger positions in asset 1 . As a result of the general alpha decay and transaction costs, the current aim portfolio has smaller positions than the Markowitz portfolio and, as a result of the differential alpha decay, the aim portfolio loads more on asset 1 . The optimal new position is found by moving partially towards the aim portfolio. Panel B shows that the expected next trade is towards the new aim, using the same logic as before. Panel $\mathrm{C}$ traces out the entire path of expected future positions. The optimal strategy is to chase a moving target, adjusting the aim for alpha decay and trading patiently by always edging partially towards the aim. ${ }^{7}$ 
To further understand the aim portfolio, we can characterize the effect of the future expected Markowitz portfolios in terms of the different trading signals (or factors), $f_{t}$, and their mean reversion speeds. Naturally, a more persistent factor has a larger effect on future Markowitz portfolios than a factor that quickly mean reverts. Indeed, the central relevance of signal persistence in the presence of transaction costs is one of the distinguishing features of our analysis.

Proposition 4 (Weight Signals Based on Alpha Decay) (i) Under Assumption A, the aim portfolio is the Markowitz portfolio built as if the signals $f$ were scaled down based on their mean reversion $\Phi$ :

$$
\operatorname{aim}_{t}=(\gamma \Sigma)^{-1} B\left(I+\frac{a}{\gamma} \Phi\right)^{-1} f_{t}
$$

(ii) If the matrix $\Phi$ is diagonal, $\Phi=\operatorname{diag}\left(\phi^{1}, \ldots, \phi^{K}\right)$, then the aim portfolio simplifies as the Markowitz portfolio with each factor $f_{t}^{k}$ scaled down based on its own alpha decay $\phi^{k}$ :

$$
\operatorname{aim}_{t}=(\gamma \Sigma)^{-1} B\left(\frac{f_{t}^{1}}{1+\phi^{1} a / \gamma}, \ldots, \frac{f_{t}^{K}}{1+\phi^{K} a / \gamma}\right)^{\top}
$$

(iii) A persistent factor $i$ is scaled down less than a fast factor $j$, and the relative weight of $i$ compared to that of $j$ increases in the transaction cost, i.e., $\left(1+\phi^{j} a / \gamma\right) /\left(1+\phi^{i} a / \gamma\right)$ increases in $\lambda$.

This proposition shows explicitly the close link between the optimal dynamic aim portfolio in light of transaction costs and the classic Markowitz portfolio. The aim portfolio resembles the Markowitz portfolio, but the factors are scaled down based on transaction costs (captured by $a$ ), risk aversion $(\gamma)$, and, importantly, the mean-reversion speed of the factors $(\Phi)$.

The aim portfolio is particularly simple under the rather standard assumption that the dynamics of each factor $f^{k}$ depend only on its own level (not the level of the other factors), that is, $\Phi=\operatorname{diag}\left(\phi^{1}, \ldots, \phi^{K}\right)$ is diagonal, so that Equation (2) simplifies to scalars:

$$
\Delta f_{t+1}^{k}=-\phi^{k} f_{t}^{k}+\varepsilon_{t+1}^{k}
$$


The resulting aim portfolio is very similar to the Markowitz portfolio, $(\gamma \Sigma)^{-1} B f_{t}$. Hence, transaction costs imply first that one optimally only trades part of the way towards the aim, and, second, that the aim down-weights each return-predicting factor more the higher is its alpha decay $\phi^{k}$. Down-weighting factors reduces the size of the position, and, more importantly, changes the relative importance of the different factors. This feature is also seen in Figure 2. The convex J-shape of the path of expected future Markowitz portfolios indicates that the factors that predict a high return for asset 2 decay faster than those that predict asset 1. To make this point in a different way, if the expected returns of the two assets decayed equally fast, then the Markowitz portfolio would be expected to move linearly towards its long-term mean. Since the aim portfolio downweights the faster decaying factors, the investor trades less towards asset 2. To see this graphically, note that the aim lies below the line joining the Markowitz portfolio with the origin, thus downweighting asset 2 relative to asset 1. Naturally, giving more weight to the more persistent factors means that the investor trades towards a portfolio that not only has a high expected return now, but also is expected to have a high expected return for a longer time in the future.

We end this section by considering what portfolio an investor ends up owning if he always follows our optimal strategy.

Proposition 5 (Position Homing In) Suppose that the agent has followed the optimal trading strategy from time $-\infty$ until time $t$. Then the current portfolio is an exponentially weighted average of past aim portfolios. Under Assumption A,

$$
x_{t}=\sum_{\tau=-\infty}^{t} \frac{a}{\lambda}\left(1-\frac{a}{\lambda}\right)^{t-\tau} \operatorname{aim}_{\tau}
$$

We see that the optimal portfolio is an exponentially weighted average of current and past aim portfolios. Clearly, the history of the past expected returns affects the current position, since the investor trades patiently to economize on transaction costs. One reading of the proposition is that the investor computes the exponentially weighted average of past aim portfolios and always trades all the way to this portfolio (assuming that his initial portfolio is right, otherwise the first trade is suboptimal). 


\section{Persistent Transaction Costs}

In some cases the impact of trading on prices may have a non-negligible persistent component. If an investor trades weekly and the current prices are unaffected by his trades during the previous week, then the temporary transaction cost model above is appropriate. However, if the frequency of trading is large relative to the the resiliency of prices, then the investor will be affected by persistent price-impact costs.

To study this situation, we extend the model by letting the price be given by $\bar{p}_{t}=p_{t}+D_{t}$ and the investor incur the cost associated with the persistent price distortion $D_{t}$ in addition to the temporary trading cost $T C$ from before. Hence, the price $\bar{p}_{t}$ is the sum of the price $p_{t}$ without the persistent effect of the investor's own trading (as before) and the new term $D_{t}$, which captures the accumulated price distortion due to the investor's (previous) trades. Trading an amount $\Delta x_{t}$ pushes prices by $C \Delta x_{t}$ such that the price distortion becomes $D_{t}+C \Delta x_{t}$, where $C$ is Kyle's lambda for persistent price moves. Further, the price distortion mean reverts at a speed (or "resiliency") $R$. Hence, the price distortion next period $(t+1)$ is

$$
D_{t+1}=(I-R)\left(D_{t}+C \Delta x_{t}\right)
$$

The investor's objective is as before, with a natural modification due to the price distortion:

$$
\begin{gathered}
E_{0}\left[\sum_{t}(1-\rho)^{t+1}\left(x_{t}^{\top}\left[B f_{t}-\left(R+r^{f}\right)\left(D_{t}+C \Delta x_{t}\right)\right]-\frac{\gamma}{2} x_{t}^{\top} \Sigma x_{t}\right)\right. \\
\left.\quad+(1-\rho)^{t}\left(-\frac{1}{2} \Delta x_{t}^{\top} \Lambda \Delta x_{t}+x_{t-1}^{\top} C \Delta x_{t}+\frac{1}{2} \Delta x_{t}^{\top} C \Delta x_{t}\right)\right] .
\end{gathered}
$$

Let us explain the various new terms in this objective function. The first term is the position $x_{t}$ times the expected excess return of the price $\bar{p}_{t}=p_{t}+D_{t}$ given inside the inner square brackets. As before, the expected excess return of $p_{t}$ is $B f_{t}$. The expected excess return due 
to the post-trade price distortion is

$$
D_{t+1}-\left(1+r^{f}\right)\left(D_{t}+C \Delta x_{t}\right)=-\left(R+r^{f}\right)\left(D_{t}+C \Delta x_{t}\right) \text {. }
$$

The second term is the penalty for taking risk as before. The three terms on the second line of (19) are discounted at $(1-\rho)^{t}$ because these cash flows are incurred at time $t$, not time $t+1$. The first of these is the temporary transaction cost as before. The second reflects the mark-to-market gain from the old position $x_{t-1}$ from the price impact of the new trade, $C \Delta x_{t}$. The last term reflects that the traded shares $\Delta x_{t}$ are assumed to be executed at the average price distortion, $D_{t}+\frac{1}{2} C \Delta x_{t}$. Hence, the traded shares $\Delta x_{t}$ earn a mark-to-market gain of $\frac{1}{2} \Delta x_{t}^{\top} C \Delta x_{t}$ as the price moves up an additional $\frac{1}{2} C \Delta x_{t}$.

The value function is now quadratic in the extended state variable $\left(x_{t-1}, y_{t}\right) \equiv\left(x_{t-1}, f_{t}, D_{t}\right)$ :

$$
V(x, y)=-\frac{1}{2} x^{\top} A_{x x} x+x^{\top} A_{x y} y+\frac{1}{2} y^{\top} A_{y y} y+A_{0}
$$

As before, there exists a unique solution to the Bellman equation and the following proposition characterizes the optimal portfolio strategy.

Proposition 6 The optimal portfolio $x_{t}$ is

$$
x_{t}=x_{t-1}+M^{\text {rate }}\left(\text { aim }_{t}-x_{t-1}\right) \text {, }
$$

which tracks an aim portfolio, aim ${ }_{t}=M^{a i m} y_{t}$. The aim portfolio depends on the returnpredicting factors and the price distortion, $y_{t}=\left(f_{t}, D_{t}\right)$. The coefficient matrices $M^{\text {rate }}$ and $M^{\text {aim }}$ are stated in the appendix.

The optimal trading policy has a similar structure to before, but the persistent price impact changes both the trading rate and the aim portfolio. The aim is now a weighted average of current and expected future Markowitz portfolios, as well as the current price distortion.

Figure 3 illustrates graphically the optimal trading strategy with temporary and persistent price impact. Panel A uses the parameters from Figures 1-2 with only temporary [Figure 3] transaction costs, Panel B has both temporary and persistent transaction costs, while Panel 
C has purely persistent price impact. Specifically, suppose that Kyle's lambda for temporary price impact is $\Lambda=w \tilde{\Lambda}$ and Kyle's lambda for persistent price impact is $C=(1-w) \tilde{\Lambda}$, where we vary $w$ to determine how much of the price impact is temporary versus persistent and where $\tilde{\Lambda}$ is a fixed matrix. Panel A has $w=1$ (pure temporary costs), Panel B has $w=0.5$ (both temporary and persistent costs), and Panel C has $w=0$ (pure persistent costs). ${ }^{8}$

We see that the optimal portfolio policy with persistent transaction costs also tracks the Markowitz portfolio while aiming in front of the target. It can be shown more generally that the optimal portfolio under persistent price impact depends on the expected future Markowitz portfolios (i.e., aims in front of the target). This is similar to the case of temporary price impact, but what is different with purely persistent price impact is that the initial trade is larger and, even in continuous time, there can be jumps in the portfolio. This is because, when the price impact is persistent, the trader incurs a transaction cost based on the entire cumulative trade, and therefore is more willing to incur it early in order to start collecting the benefits of a better portfolio. (The resilience still makes it cheaper to postpone part of trade, however). Furthermore, the cost of buying a position and selling it shortly thereafter is much smaller with persistent price impact.

\section{Theoretical Applications}

We next provide a few simple and useful examples of our model.

\section{Example 1: Timing a single security}

A simple case is when there is only one security. This occurs when an investor is timing his long or short view of a particular security or market. In this case, Assumption $\mathrm{A}(\Lambda=\lambda \Sigma)$ is without loss of generality since all parameters are scalars, and we use the notation $\sigma^{2}=\Sigma$

and $B=\left(\beta^{1}, \ldots, \beta^{K}\right)$. Assuming that $\Phi$ is diagonal, we can apply Proposition 4 directly to 
get the optimal timing portfolio:

$$
x_{t}=\left(1-\frac{a}{\lambda}\right) x_{t-1}+\frac{a}{\lambda} \frac{1}{\gamma \sigma^{2}} \sum_{i=1}^{K} \frac{\beta^{i}}{1+\phi^{i} a / \gamma} f_{t}^{i} .
$$

\section{Example 2: Relative-value trades based on security characteristics}

It is natural to assume that the agent uses certain characteristics of each security to predict its returns. Hence, each security has its own return-predicting factors (whereas, in the general model above, all the factors could influence all the securities). For instance, one can imagine that each security is associated with a value characteristic (e.g., its own book-to-market) and a momentum characteristic (its own past return). In this case, it is natural to let the expected return for security $s$ be given by

$$
E_{t}\left(r_{t+1}^{s}\right)=\sum_{i} \beta^{i} f_{t}^{i, s}
$$

where $f_{t}^{i, s}$ is characteristic $i$ for security $s$ (e.g., IBM's book-to-market) and $\beta^{i}$ be the predictive ability of characteristic $i$ (i.e., how book-to-market translates into future expected return, for any security), which is the same for all securities $s$. Further, we assume that characteristic $i$ has the same mean-reversion speed for each security, that is, for all $s$,

$$
\Delta f_{t+1}^{i, s}=-\phi^{i} f_{t}^{i, s}+\varepsilon_{t+1}^{i, s} .
$$

We collect the current values of characteristic $i$ for all securities in a vector $f_{t}^{i}=\left(f_{t}^{i, 1}, \ldots, f_{t}^{i, S}\right)^{\top}$, e.g., the book-to-market of security 1, book-to-market of security 2 , etc.

This setup based on security characteristics is a special case of our general model. To map it into the general model, we stack all the various characteristic vectors on top of each 
other into $f$ :

$$
f_{t}=\left(\begin{array}{c}
f_{t}^{1} \\
\vdots \\
f_{t}^{I}
\end{array}\right)
$$

Further, we let $I_{S \times S}$ be the $S$-by-S identity matrix and can express $B$ using the Kronecker product:

$$
B=\beta^{\top} \otimes I_{S \times S}=\left(\begin{array}{ccccccc}
\beta^{1} & 0 & 0 & & \beta^{I} & 0 & 0 \\
0 & \ddots & 0 & \ldots & 0 & \ddots & 0 \\
0 & 0 & \beta^{1} & & 0 & 0 & \beta^{I}
\end{array}\right)
$$

Thus, $E_{t}\left(r_{t+1}\right)=B f_{t}$. Also, let $\Phi=\operatorname{diag}\left(\phi \otimes 1_{S \times 1}\right)=\operatorname{diag}\left(\phi^{1}, \ldots, \phi^{1}, \ldots, \phi^{I}, \ldots, \phi^{I}\right)$. With these definitions, we apply Proposition 4 to get the optimal characteristic-based relativevalue trade as

$$
x_{t}=\left(1-\frac{a}{\lambda}\right) x_{t-1}+\frac{a}{\lambda}(\gamma \Sigma)^{-1} \sum_{i=1}^{I} \frac{1}{1+\phi^{i} a / \gamma} \beta^{i} f_{t}^{i} .
$$

\section{Example 3: Static model}

Consider an investor who performs a static optimization involving current expected returns, risk, and transaction costs. Such an investor simply solves

$$
\max _{x_{t}} x_{t}^{\top} E_{t}\left(r_{t+1}\right)-\frac{\gamma}{2} x_{t}^{\top} \Sigma x_{t}-\frac{\lambda}{2} \Delta x_{t}^{\top} \Sigma \Delta x_{t}
$$

with solution

$$
x_{t}=\frac{\lambda}{\gamma+\lambda} x_{t-1}+\frac{\gamma}{\gamma+\lambda}(\gamma \Sigma)^{-1} E_{t}\left(r_{t+1}\right)=x_{t-1}+\frac{\gamma}{\gamma+\lambda}\left(\text { Markowitz }_{t}-x_{t-1}\right) .
$$

This optimal static portfolio in light of transaction costs differs from our optimal dynamic portfolio in two ways: (i) The weight on the current portfolio $x_{t-1}$ is different, and (ii) the 
aim portfolio is different since in the static case the aim portfolio is the Markowitz portfolio. Problem (i) with the static portfolio, namely that it prescribes a suboptimal trading rate because it does not account for the future benefits of the position can be fixed by changing the transaction-cost parameter $\lambda$ (or risk aversion $\gamma$ or both).

However, problem (ii) cannot be fixed in this way. Interestingly, with multiple returnpredicting factors, no choice of risk aversion $\gamma$ and trading cost $\lambda$ recovers the dynamic solution. This is because the static solution treats all factors the same, while the dynamic solution gives more weight to factors with slower alpha decay. We show empirically in Section VI that even the best choice of $\gamma$ and $\lambda$ in a static model may perform significantly worse than our dynamic solution. To recover the dynamic solution in a static setting, one must change not just $\gamma$ and $\lambda$, but additionally the expected returns $E_{t}\left(r_{t+1}\right)=B f_{t}$ by changing $B$ as described in Proposition 4.

\section{Example 4: Today's first signal is tomorrow's second signal}

Suppose that the investor is timing a single market using each of the several past daily returns to predict the next return. In other words, the first signal $f_{t}^{1}$ is the daily return for yesterday, the second signal $f_{t}^{2}$ is the return the day before yesterday, and so on for $K$ past time periods. In this case, the trader already knows today what some of her signals will look like in the future. Today's yesterday is tomorrow's day-before-yesterday:

$$
\begin{aligned}
& f_{t+1}^{1}=\varepsilon_{t+1}^{1} \\
& f_{t+1}^{k}=f_{t}^{k-1} \quad \text { for } k>1
\end{aligned}
$$

Said differently, the matrix $\Phi$ has the form

$$
I-\Phi=\left(\begin{array}{cccc}
0 & & & 0 \\
1 & 0 & & \\
& \ddots & \ddots & \\
0 & & 1 & 0
\end{array}\right)
$$


Suppose for simplicity that all signals are equally important for predicting returns $B=$ $(\beta, \ldots, \beta)$ and use the notation $\sigma^{2}=\Sigma$. Then we can use Proposition 4 to get the optimal trading strategy

$$
\begin{aligned}
x_{t} & =\left(1-\frac{a}{\lambda}\right) x_{t-1}+\frac{a}{\lambda} \frac{1}{\sigma^{2}} B(\gamma+a \Phi)^{-1} f_{t} \\
& =\left(1-\frac{a}{\lambda}\right) x_{t-1}+\frac{a}{\lambda} \frac{\beta}{\gamma \sigma^{2}} \sum_{k=1}^{K}\left(1-\left(\frac{a}{\gamma+a}\right)^{K+1-k}\right) f_{t}^{k} .
\end{aligned}
$$

Hence, the optimal portfolio gives the largest weight to the first signal (yesterday's return), the second largest to the second signal, and so on. This is intuitive, since the first signal will continue to be important the longest, the second signal the second longest, and so on.

\section{Empirical Application: Dynamic Trading of Com- modity Futures}

In this section we illustrate our approach using data on commodity futures. We show how dynamic optimizing can improve performance in an intuitive way, and how it changes the way new information is used.

\section{A. Data}

We consider 15 different liquid commodity futures, which do not have tight restrictions on the size of daily price moves (limit up/down). In particular, as seen in Table I, we collect data on Aluminum, Copper, Nickel, Zinc, Lead, and Tin from the London Metal Exchange (LME), Gasoil from the Intercontinental Exchange (ICE), WTI Crude, RBOB Unleaded Gasoline, and Natural Gas from the New York Mercantile Exchange (NYMEX), Gold and Silver from the New York Commodities Exchange (COMEX), and Coffee, Cocoa, and Sugar from the New York Board of Trade (NYBOT). (This excludes futures on various agriculture

and livestock that have tight price limits.) We consider the sample period 01/01/1996 $01 / 23 / 2009$, for which we have data on all the commodities. ${ }^{9}$ 
For each commodity and each day, we collect the futures price measured in U.S. dollars per contract. For instance, if the gold price is $\$ 1,000$ per ounce, the price per contract is $\$ 100,000$, since each contract is for 100 ounces. Table I provides summary statistics on each contract's average price, the standard deviation of price changes, the contract multiplier (e.g., 100 ounces per contract in the case of gold), and daily trading volume.

We use the most liquid futures contract of all maturities available. By always using data on the most liquid futures, we are implicitly assuming that the trader's position is always held in these contracts. Hence, we are assuming that when the most liquid futures nears maturity and the next contract becomes more liquid, the trader "rolls" into the next contract, i.e., replaces the position in the near contract with the same position in the far contract. Given that rolling does not change a trader's net exposure, it is reasonable to abstract from the transaction costs associated with rolling. (Traders in the real world do in fact behave like this. There is a separate roll market, which entails far smaller costs than independently selling the "old" contract and buying the "new" one.) When we compute price changes, we always compute the change in price of a given contract (not the difference between the new contract and the old one), since this corresponds to an implementable return. Finally, we collect data on the average daily trading volume per contract as seen in the last column of Table I. Specifically, we receive an estimate of the average daily volume of the most liquid contract traded electronically and outright (i.e., not including calendar-spread trades) in December 2010 from an asset manager based on underlying data from Reuters.

\section{B. Predicting Returns and Other Parameter Estimates}

We use the characteristic-based model described in Example 2 in Section II, where each commodity characteristic is its own past return at various horizons. Hence, to predict returns, we run a pooled panel regression:

$$
r_{t+1}^{s}=0.001+10.32 f_{t}^{5 D, s}+122.34 f_{t}^{1 Y, s}-205.59 f_{t}^{5 Y, s}+u_{t+1}^{s}
$$


where the left-hand side is the daily commodity price changes and the right-hand side contains the return predictors: $f^{5 D}$ is the average past five days' price change, divided by the past five days' standard deviation of daily price changes, $f^{1 Y}$ is the past year's average daily price change divided by the past year's standard deviation, and $f^{5 Y}$ is the analogous quantity for a five-year window. Hence, the predictors are rolling Sharpe ratios over three different horizons, and, to avoid dividing by a number close to zero, the standard deviations are winsorized below the tenth percentile of standard deviations. We estimate the regression using feasible generalized least squares and report the t-statistics in brackets.

We see that price changes show continuation at short and medium frequencies and reversal over long horizons. ${ }^{10}$ The goal is to see how an investor could optimally trade on this information, taking transaction costs into account. Of course, these (in-sample) regression results are only available now and a more realistic analysis would consider rolling out-of-sample regressions. However, using the in-sample regression allows us to focus on the economic insights underlying our novel portfolio optimization. Indeed, the in-sample analysis allows us to focus on the benefits of giving more weight to signals with slower alpha decay, without the added noise in the predictive power of the signals arising when using out-of-sample return forecasts.)

The return predictors are chosen so that they have very different mean reversion:

$$
\begin{aligned}
\Delta f_{t+1}^{5 D, s} & =-0.2519 f_{t}^{5 D, s}+\varepsilon_{t+1}^{5 D, s} \\
\Delta f_{t+1}^{1 Y, s} & =-0.0034 f_{t}^{1 Y, s}+\varepsilon_{t+1}^{1 Y, s} \\
\Delta f_{t+1}^{5 Y, s} & =-0.0010 f_{t}^{5 Y, s}+\varepsilon_{t+1}^{5 Y, s} .
\end{aligned}
$$

These mean reversion rates correspond to a 2.4-day half life for the five-day signal, a 206-day half life for the one-year signal, and a 700-day half life for the five-year signal. ${ }^{11}$

We estimate the variance-covariance matrix $\Sigma$ using daily price changes over the full sample, shrinking the correlations $50 \%$ towards zero. We set the absolute risk aversion to $\gamma=10^{-9}$, which we can think of as corresponding to a relative risk aversion of 1 for an agent with 1 billion dollars under management. We set the time discount rate to $\rho=$ 
$1-\exp (-0.02 / 260)$ corresponding to a 2 percent annualized rate.

Finally, to choose the transaction-cost matrix $\Lambda$, we make use of price-impact estimates from the literature. In particular, we use the estimate from Engle, Ferstenberg, and Russell (2008) that trades amounting to $1.59 \%$ of the daily volume in a stock have a price impact of about $0.10 \%$. (Breen, Hodrick, and Korajczyk (2002) provides a similar estimate.) Further, Greenwood (2005) finds evidence that market impact in one security spills over to other securities using the specification $\Lambda=\lambda \Sigma$, where we recall that $\Sigma$ is the variance-covariance matrix. We calibrate $\Sigma$ as the empirical variance-covariance matrix of price changes, where the covariance are shrunk $50 \%$ towards zero for robustness.

We choose the scalar $\lambda$ based on the Engle, Ferstenberg, and Russell (2008) estimate by calibrating it for each commodity and then computing the mean and median across commodities. Specifically, we collect data on the trading volume of each commodity contract as seen in last column of Table I and then calibrate $\lambda$ for each commodity as follows. Consider, for instance, unleaded gasoline. Since gasoline has a turnover of 11,320 contracts per day and a daily price-change volatility of $\$ 1,340$, the transaction cost per contract when one trades $1.59 \%$ of daily volume is $1.59 \% \times 11,320 \times \lambda^{\text {Gasoline }} / 2 \times 1,340^{2}$, which is $0.10 \%$ of the average price per contract of $\$ 48,000$ if $\lambda^{\text {Gasoline }}=3 \times 10^{-7}$.

We calibrate the trading costs for the other commodities similarly, and obtain a median value of $5.0 \times 10^{-7}$ and a mean of $8.4 \times 10^{-7}$. There are significant differences across commodities (e.g., the standard deviation is $1.0 \times 10^{-6}$ ), reflecting that these estimates are based on turnover while the specification $\Lambda=\lambda \Sigma$ assumes that transaction costs depend on variances. While our model is general enough to handle transaction costs that depend on turnover (e.g., by using these calibrated $\lambda$ 's in the diagonal of the $\Lambda$ matrix), we also need to estimate the spill-over effects (i.e., the off-diagonal elements). Since Greenwood (2005) provides the only estimate of these transaction-cost spill-overs in the literature using the assumption $\Lambda=\lambda \Sigma$ and since real-world transaction costs likely depend on variance as well as turnover, we stick to this specification and calibrate $\lambda$ as the median across the estimates for each commodity. Naturally, other specifications of the transaction-cost matrix would give 
slightly different results, but our main purpose is simply to illustrate the economic insights that we have proved in general theoretically.

We also consider a more conservative transaction cost estimate of $\lambda=10 \times 10^{-7}$. Alternatively, this more conservative analysis can be interpreted as the trading strategy of a larger investor (i.e., we could have equivalently reduced the absolute risk aversion $\gamma$ ).

\section{Dynamic Portfolio Selection with Trading Costs}

We consider three different trading strategies: the optimal trading strategy given by Equation (27) ("optimal"), the optimal trading strategy in the absence of transaction costs ("Markowitz"), and a number of trading strategies based on a static (i.e., one-period) transaction-cost optimization as in Equation (29) ("static optimization"). The static portfolio optimization results in trading partially towards the Markowitz portfolio (as opposed to an aim portfolio that depends on signals' alpha decays) and we consider ten different trading speeds in seen in Table II. Hence, under the static optimization, the updated portfolio is a weighted average of the Markowitz portfolio (with weight denoted "weight on Markowitz") and the current portfolio.

Table II reports the performance of each strategy as measured by, respectively, its Gross Sharpe Ratio and its Net Sharpe Ratio (i.e., its Sharpe ratio after accounting for transaction costs). Panel A reports these numbers using our base-case transaction-cost estimate (discussed above), while Panel B uses our high transaction-cost estimate. We see that, naturally, the highest SR before transaction costs is achieved by the Markowitz strategy. The optimal and static portfolios have similar drops in gross SR due to their slower trading. After transaction costs, however, the optimal portfolio is the best, significantly better than the best possible static strategy, and the Markowitz strategy incurs enormous trading costs.

It is interesting to consider the driver of the superior performance of the optimal dynamic trading strategy relative to the best possible static strategy. The key to the out-performance is that the dynamic strategy gives less weight to the five-day signal because of its fast alpha decay. The static strategy simply tries to control the overall trading speed, but this is not 
sufficient: it either incurs large trading costs due to its "fleeting" target (because of the significant reliance on the five-day signal), or it trades so slowly it is difficult to capture the return. The dynamic strategy overcomes this problem by trading somewhat fast, but trading mainly according to the more persistent signals.

To illustrate the difference in the positions of the different strategies, Figure 4 shows

the positions over time of two of the commodity futures, namely Crude and Gold. We see that the optimal portfolio is a much smoother version of the Markowitz strategy, thus reducing trading costs while at the same time capturing most of the excess return. Indeed, the optimal position tends to be long when the Markowitz portfolio is long and short when the Markowitz portfolio is short, and to be larger when the expected return is large, but moderates the speed and magnitude of trades.

\section{Response to New Information}

It is instructive to trace the response to a shock to the return predictors, namely to $\varepsilon_{t}^{i, s}$ in Equation (32). Figure 5 shows the responses to shocks to each return-predicting factor, [Figure 5] namely the five-day factor, the one-year factor, and the five-year factor.

The first panel shows that the Markowitz strategy immediately jumps up after a shock to the five-day factor and slowly mean reverts as the alpha decays. The optimal strategy trades much more slowly and never accumulates nearly as large a position. Interestingly, since the optimal position also trades more slowly out of the position as the alpha decays, the lines cross as the optimal strategy eventually has a larger position than the Markowitz strategy.

The second panel shows the response to the one-year factor. The Markowitz jumps up and decays, whereas the optimal position increases more smoothly and catches up as the Markowitz starts to decay. The third panel shows the same for the $5 \mathrm{Y}$ signal, except that the effects are slower and with opposite sign, since five-year returns predict future reversals. 


\section{Conclusion}

This paper provides a highly tractable framework for studying optimal trading strategies in the presence of several return predictors, risk and correlation considerations, as well as transaction costs. We derive an explicit closed-form solution for the optimal trading policy, which gives rise to several intuitive results. The optimal portfolio tracks an "aim portfolio," which is analogous to the optimal portfolio in the absence of trading costs in its trade-off between risk and return, but different since more persistent return predictors are weighted more heavily relative to return predictors with faster alpha decay. The optimal strategy is not to trade all the way to the aim portfolio, since this entails too high transaction costs. Instead, it is optimal to take a smoother and more conservative portfolio that moves in the direction of the aim portfolio while limiting turnover.

Our framework constitutes a powerful tool to optimally combine various return predictors taking into account their evolution over time, decay rate, and correlation, and trading off their benefits against risks and transaction costs. Such dynamic trade-offs are at the heart of the decisions of "arbitrageurs" that help make markets efficient as per the efficient market hypothesis. Arbitrageurs' ability to do so is limited, however, by transaction costs, and our model provides a tractable and flexible framework for the study of the dynamic implications of this limitation.

We implement our optimal trading strategy for commodity futures. Naturally, the optimal trading strategy in the absence of transaction costs has a larger Sharpe ratio gross of fees than our trading policy. However, net of trading costs our strategy performs significantly better, since it incurs far lower trading costs while still capturing much of the return predictability and diversification benefits. Further, the optimal dynamic strategy is significantly better than the best static strategy — i.e., taking dynamics into account significantly improves performance.

In conclusion, we provide a tractable solution to the dynamic trading strategy in a relevant and general setting that we believe to have many interesting applications. The main insights for portfolio selection can be summarized by the rules that one should aim in front 
of the target and trade partially towards the current aim. 


\section{A. Proofs}

In what follows we'll make repeated use of the notation

$$
\begin{aligned}
& \bar{\rho}=1-\rho \\
& \bar{\Lambda}=\bar{\rho}^{-1} \Lambda \\
& \bar{\lambda}=\bar{\rho}^{-1} \lambda .
\end{aligned}
$$

Proof of Proposition 1. Assuming that the value function is of the posited form, we calculate the expected future value function as

$$
\begin{aligned}
E_{t}\left[V\left(x_{t}, f_{t+1}\right)\right]= & -\frac{1}{2} x_{t}^{\top} A_{x x} x_{t}+x_{t}^{\top} A_{x f}(I-\Phi) f_{t}+\frac{1}{2} f_{t}^{\top}(I-\Phi)^{\top} A_{f f}(I-\Phi) f_{t} \\
& +\frac{1}{2} E_{t}\left(\varepsilon_{t+1}^{\top} A_{f f} \varepsilon_{t+1}\right)+A_{0} .
\end{aligned}
$$

The agent maximizes the quadratic objective $-\frac{1}{2} x_{t}^{\top} J_{t} x_{t}+x_{t}^{\top} j_{t}+d_{t}$ with

$$
\begin{aligned}
J_{t} & =\gamma \Sigma+\bar{\Lambda}+A_{x x} \\
j_{t} & =\left(B+A_{x f}(I-\Phi)\right) f_{t}+\bar{\Lambda} x_{t-1} \\
d_{t} & =-\frac{1}{2} x_{t-1}^{\top} \bar{\Lambda} x_{t-1}+\frac{1}{2} f_{t}^{\top}(I-\Phi)^{\top} A_{f f}(I-\Phi) f_{t}+\frac{1}{2} E_{t}\left(\varepsilon_{t+1}^{\top} A_{f f} \varepsilon_{t+1}\right)+A_{0} .
\end{aligned}
$$

The maximum value is attained by

$$
x_{t}=J_{t}^{-1} j_{t},
$$

and it is equal to $V\left(x_{t-1}, f_{t}\right)=\frac{1}{2} j_{t}^{\top} J_{t}^{-1} j_{t}+d_{t}$. Combining this fact with (6) we obtain an equation that must hold for all $x_{t-1}$ and $f_{t}$, which implies the following restrictions on the 
coefficient matrices: ${ }^{12}$

$$
\begin{aligned}
-\bar{\rho}^{-1} A_{x x}= & \bar{\Lambda}\left(\gamma \Sigma+\bar{\Lambda}+A_{x x}\right)^{-1} \bar{\Lambda}-\bar{\Lambda} \\
\bar{\rho}^{-1} A_{x f}= & \bar{\Lambda}\left(\gamma \Sigma+\bar{\Lambda}+A_{x x}\right)^{-1}\left(B+A_{x f}(I-\Phi)\right) \\
\bar{\rho}^{-1} A_{f f}= & \left(B+A_{x f}(I-\Phi)\right)^{\top}\left(\gamma \Sigma+\bar{\Lambda}+A_{x x}\right)^{-1}\left(B+A_{x f}(I-\Phi)\right) \\
& +(I-\Phi)^{\top} A_{f f}(I-\Phi) .
\end{aligned}
$$

The existence of a solution to this system of Riccati equations can be established using standard results, e.g., as in Ljungqvist and Sargent (2004). In this case, however, we can derive explicit expressions, as follows. We start by letting $Z=\bar{\Lambda}^{-\frac{1}{2}} A_{x x} \bar{\Lambda}^{-\frac{1}{2}}$ and $M=$ $\bar{\Lambda}^{-\frac{1}{2}} \Sigma \bar{\Lambda}^{-\frac{1}{2}}$ and rewriting Equation (A.7) as

$$
\bar{\rho}^{-1} Z=I-(\gamma M+I+Z)^{-1}
$$

which is a quadratic with an explicit solution. Since all solutions $Z$ can be written as a limit of polynomials of $M, Z$ and $M$ commute and the quadratic can be sequentially rewritten as

$$
\begin{aligned}
Z^{2}+Z(I+\gamma M-\bar{\rho} I) & =\bar{\rho} \gamma M \\
\left(Z+\frac{1}{2}(\gamma M+\rho I)\right)^{2} & =\bar{\rho} \gamma M+\frac{1}{4}(\gamma M+\rho I)^{2},
\end{aligned}
$$

resulting in

$$
\begin{aligned}
Z & =\left(\bar{\rho} \gamma M+\frac{1}{4}(\rho I+\gamma M)^{2}\right)^{\frac{1}{2}}-\frac{1}{2}(\rho I+\gamma M) \\
A_{x x} & =\bar{\Lambda}^{\frac{1}{2}}\left[\left(\bar{\rho} \gamma M+\frac{1}{4}(\rho I+\gamma M)^{2}\right)^{\frac{1}{2}}-\frac{1}{2}(\rho I+\gamma M)\right] \bar{\Lambda}^{\frac{1}{2}},
\end{aligned}
$$

that is,

$$
A_{x x}=\left(\bar{\rho} \gamma \bar{\Lambda}^{\frac{1}{2}} \Sigma \bar{\Lambda}^{\frac{1}{2}}+\frac{1}{4}\left(\rho^{2} \bar{\Lambda}^{2}+2 \rho \gamma \bar{\Lambda}^{\frac{1}{2}} \Sigma \bar{\Lambda}^{\frac{1}{2}}+\gamma^{2} \bar{\Lambda}^{\frac{1}{2}} \Sigma \bar{\Lambda}^{-1} \Sigma \bar{\Lambda}^{\frac{1}{2}}\right)\right)^{\frac{1}{2}}-\frac{1}{2}(\rho \bar{\Lambda}+\gamma \Sigma) .
$$

Note that the positive definite choice of solution $Z$ is the only one that results in a positive 
definite matrix $A_{x x}$.

The other value-function coefficient determining optimal trading is $A_{x f}$, which solves the linear equation (A.8). To write the solution explicitly, we note first that, from (A.7),

$$
\bar{\Lambda}\left(\gamma \Sigma+\bar{\Lambda}+A_{x x}\right)^{-1}=I-A_{x x} \Lambda^{-1} .
$$

Using the general rule that $\operatorname{vec}(X Y Z)=\left(Z^{\top} \otimes X\right) \operatorname{vec}(Y)$, we rewrite (A.8) in vectorized form:

$$
\begin{aligned}
\operatorname{vec}\left(A_{x f}\right)= & \bar{\rho} \operatorname{vec}\left(\left(I-A_{x x} \Lambda^{-1}\right) B\right) \\
& +\bar{\rho}\left((I-\Phi)^{\top} \otimes\left(I-A_{x x} \Lambda^{-1}\right)\right) \operatorname{vec}\left(A_{x f}\right),
\end{aligned}
$$

so that

$$
\operatorname{vec}\left(A_{x f}\right)=\bar{\rho}\left(I-\bar{\rho}(I-\Phi)^{\top} \otimes\left(I-A_{x x} \Lambda^{-1}\right)\right)^{-1} \operatorname{vec}\left(\left(I-A_{x x} \Lambda^{-1}\right) B\right) .
$$

Finally, $A_{f f}$ is calculated from the linear equation (A.9), which is of the form

$$
\bar{\rho}^{-1} A_{f f}=Q+(I-\Phi)^{\top} A_{f f}(I-\Phi)
$$

with

$$
Q=\left(B+A_{x f}(I-\Phi)\right)^{\top}\left(\gamma \Sigma+\bar{\Lambda}+A_{x x}\right)^{-1}\left(B+A_{x f}(I-\Phi)\right)
$$

a positive-definite matrix.

The solution is easiest to write explicitly for diagonal $\Phi$, in which case

$$
A_{f f, i j}=\frac{\bar{\rho} Q_{i j}}{1-\bar{\rho}\left(1-\Phi_{i i}\right)\left(1-\Phi_{j j}\right)} .
$$

In general,

$$
\operatorname{vec}\left(A_{f f}\right)=\bar{\rho}\left(I-\bar{\rho}(I-\Phi)^{\top} \otimes(I-\Phi)^{\top}\right)^{-1} \operatorname{vec}(Q) .
$$


One way to see that $A_{f f}$ is positive definite is to iterate (A.19) starting with $A_{f f}^{0}=0$.

We conclude that the posited value function satisfies the Bellman equation.

Proof of Proposition 2. Differentiating the Bellman equation (5) with respect to $x_{t-1}$ gives

$$
-A_{x x} x_{t-1}+A_{x f} f_{t}=\Lambda\left(x_{t}-x_{t-1}\right)
$$

which clearly implies (7) and (8).

In the case $\Lambda=\lambda \Sigma$ for some scalar $\lambda>0$, and the solution to the value-function coefficients is $A_{x x}=a \Sigma$, where $a$ solves a simplified version of (A.7):

$$
-\bar{\rho}^{-1} a=\frac{\bar{\lambda}^{2}}{\gamma+\bar{\lambda}+a}-\bar{\lambda},
$$

or

$$
a^{2}+(\gamma+\bar{\lambda} \rho) a-\lambda \gamma=0
$$

with solution

$$
a=\frac{\sqrt{(\gamma+\bar{\lambda} \rho)^{2}+4 \gamma \lambda}-(\gamma+\bar{\lambda} \rho)}{2}
$$

It follows immediately that $\Lambda^{-1} A_{x x}=a / \lambda$.

Note that $a$ is symmetric in $\left(\lambda \rho(1-\rho)^{-1}, \gamma\right)$. Consequently, $a$ increases in $\lambda$ if and only if it increases in $\gamma$. Differentiating (A.25) with respect to $\lambda$, one gets

$$
2 \frac{d a}{d \lambda}=-\bar{\rho}^{-1} \rho+\frac{1}{2} \frac{(2(\gamma+\bar{\lambda} \rho)+4 \gamma)}{\sqrt{(\gamma+\bar{\lambda} \rho)^{2}+4 \gamma \lambda}} .
$$

This expression is positive if and only if

$$
\bar{\rho}^{-2} \rho^{2}\left((\gamma+\bar{\lambda} \rho)^{2}+4 \gamma \lambda\right) \leq\left((\gamma+\bar{\lambda} \rho) \bar{\rho}^{-1} \rho+2 \gamma\right)^{2}
$$


which is verified to hold with strict inequality as long as $\bar{\rho} \gamma>0$.

Finally, note that $a / \lambda$ is increasing in $\gamma$ and homogeneous of degree zero in $(\lambda, \gamma)$, so that applying Euler's theorem for homogeneous functions gives

$$
\frac{d}{d \lambda} \frac{a}{\lambda}=-\frac{d}{d \gamma} \frac{a}{\lambda}<0
$$

Proof of Proposition 3. We show that

$$
\operatorname{aim}_{t}=\left(\gamma \Sigma+A_{x x}\right)^{-1}\left(\gamma \Sigma \times \text { Markowitz }_{t}+A_{x x} \times E_{t}\left(\text { aim }_{t+1}\right)\right)
$$

by using (8), (A.8), and (A.7) successively to write

$$
\begin{aligned}
\text { aim }_{t} & =A_{x x}^{-1} A_{x f} f_{t} \\
& =A_{x x}^{-1} \Lambda\left(\gamma \Sigma+\bar{\Lambda}+A_{x x}\right)^{-1}\left(\gamma \Sigma \times \text { Markowitz }_{t}+A_{x x} \times E_{t}\left(\text { aim }_{t+1}\right)\right) \\
& =\left(\gamma \Sigma+A_{x x}\right)^{-1}\left(\gamma \Sigma \times \text { Markowitz }_{t}+A_{x x} \times E_{t}\left(\text { aim }_{t+1}\right)\right) .
\end{aligned}
$$

To obtain the last equality, re-write (A.7) as

$$
\left(\Lambda-A_{x x}\right) \Lambda^{-1}\left(\gamma \Sigma+\bar{\Lambda}+A_{x x}\right)=\bar{\Lambda}
$$

and then further

$$
\gamma \Sigma+A_{x x}=\left(\gamma \Sigma+\bar{\Lambda}+A_{x x}\right) \Lambda^{-1} A_{x x}
$$

since $A_{x x} \Lambda^{-1} \Sigma=\Sigma \Lambda^{-1} A_{x x}$. Equation (12) follows immediately as a special case. 
For part (ii), we iterate (A.29) forward to obtain

$$
\begin{aligned}
\operatorname{aim}_{t}= & \left(\gamma \Sigma+A_{x x}\right)^{-1} \times \\
& \sum_{\tau=t}^{\infty}\left(A_{x x}\left(\gamma \Sigma+A_{x x}\right)^{-1}\right)^{(\tau-t)} \gamma \Sigma \times E_{t}\left(\text { Markowitz }_{\tau}\right),
\end{aligned}
$$

which specializes to (13). Given that $a$ increases in $\lambda, z$ decreases in $\lambda$. Furthermore, $z$ increases in $\gamma$ if and only if $a / \gamma$ decreases, which is equivalent (by symmetry) to $a / \lambda$ decreasing in $\lambda$.

Proof of Proposition 4. In the case $\Lambda=\lambda \Sigma$, equation (A.8) is solved by

$$
\begin{aligned}
A_{x f} & =\lambda B((\gamma+\bar{\lambda}+a) I-\lambda(I-\Phi))^{-1} \\
& =\lambda B((\gamma+\bar{\lambda} \rho+a) I+\lambda \Phi)^{-1} \\
& =B\left(\frac{\gamma}{a}+\Phi\right)^{-1}
\end{aligned}
$$

where the last equality uses (A.23). The aim portfolio is

$$
\operatorname{aim}_{t}=(a \Sigma)^{-1} B\left(\frac{\gamma}{a}+\Phi\right)^{-1} f_{t}
$$

which is the same as (14). Equation (15) is immediate.

For part (iii), we use the result shown above (proof of Proposition 2) that $a$ increases in $\lambda$, which implies that $\left(1+\phi^{j} a / \gamma\right) /\left(1+\phi^{i} a / \gamma\right)$ does whenever $\phi^{j}>\phi^{i}$.

Proof of Proposition 5. Rewriting (7) as

$$
x_{t}=\left(I-\Lambda^{-1} A_{x x}\right) x_{t-1}+\Lambda^{-1} A_{x x} \times \operatorname{aim}_{t}
$$

and iterating this relation backwards gives

$$
x_{t}=\sum_{\tau=-\infty}^{t}\left(I-\Lambda^{-1} A_{x x}\right)^{t-\tau} \Lambda^{-1} A_{x x} \times \operatorname{aim}_{\tau} .
$$


Proof of Proposition 6. We start by defining

$$
\begin{aligned}
& \Pi=\left[\begin{array}{ll}
\Phi & 0 \\
0 & R
\end{array}\right], \quad \tilde{C}=(1-R)\left[\begin{array}{l}
0 \\
C
\end{array}\right], \\
& \tilde{B}=\left[\begin{array}{ll}
B & -\left(R+r^{f}\right)
\end{array}\right] \text {, } \\
& \tilde{\Omega}=\left[\begin{array}{cc}
\Omega & 0 \\
0 & 0
\end{array}\right], \quad \tilde{\varepsilon}_{t}=\left[\begin{array}{c}
\varepsilon_{t} \\
0
\end{array}\right] .
\end{aligned}
$$

It is useful to keep in mind that $y_{t}=\left(f_{t}^{\top}, D_{t}^{\top}\right)^{\top}$ (a column vector). Given this definition, it follows that

$$
E_{t}\left[y_{t+1}\right]=(I-\Pi) y_{t}+\tilde{C}\left(x_{t}-x_{t-1}\right)
$$

The conjectured value function is

$$
V\left(x_{t-1}, y_{t}\right)=-\frac{1}{2} x_{t-1}^{\top} A_{x x} x_{t-1}+x_{t-1}^{\top} A_{x y} y_{t}+\frac{1}{2} y_{t}^{\top} A_{y y} y_{t}+A_{0}
$$

so that

$$
\begin{aligned}
E_{t}\left[V\left(x_{t}, y_{t+1}\right)\right]= & -\frac{1}{2} x_{t}^{\top} A_{x x} x_{t}+x_{t}^{\top} A_{x y}\left((I-\Pi) y_{t}+\tilde{C}\left(x_{t}-x_{t-1}\right)\right)+\quad(\mathrm{A} .40) \\
& \frac{1}{2}\left((I-\Pi) y_{t}+\tilde{C}\left(x_{t}-x_{t-1}\right)\right)^{\top} A_{y y}\left((I-\Pi) y_{t}+\tilde{C}\left(x_{t}-x_{t-1}\right)\right)+ \\
& \frac{1}{2} E_{t}\left[\tilde{\varepsilon}_{t+1}^{\top} A_{y y} \tilde{\varepsilon}_{t+1}\right]+A_{0} .
\end{aligned}
$$


The trader consequently chooses $x_{t}$ to solve

$$
\begin{aligned}
\max _{x}\{ & x^{\top} \tilde{B} y_{t}-x^{\top}\left(R+r^{f}\right) C\left(x-x_{t-1}\right)-\frac{\gamma}{2} x^{\top} \Sigma x \\
& +\frac{1}{2} \bar{\rho}^{-1}\left(x^{\top} C x-x_{t-1}^{\top} C x_{t-1}-\left(x-x_{t-1}\right)^{\top} \Lambda\left(x-x_{t-1}\right)\right) \\
& -\frac{1}{2} x^{\top} A_{x x} x+x^{\top} A_{x y}\left((I-\Pi) y_{t}+\tilde{C}\left(x-x_{t-1}\right)\right) \\
& \left.+\frac{1}{2}\left((I-\Pi) y_{t}+\tilde{C}\left(x-x_{t-1}\right)\right)^{\top} A_{y y}\left((I-\Pi) y_{t}+\tilde{C}\left(x-x_{t-1}\right)\right)\right\},
\end{aligned}
$$

which is a quadratic of the form $-\frac{1}{2} x^{\top} J x+x^{\top} j_{t}+d_{t}$, with

$$
\begin{aligned}
J= & \frac{1}{2}\left(J_{0}+J_{0}^{\top}\right) \\
J_{0}= & \gamma \Sigma+\bar{\Lambda}+\left(2\left(R+r^{f}\right)-\bar{\rho}^{-1}\right) C+A_{x x}-2 A_{x y} \tilde{C}-\tilde{C}^{\top} A_{y y} \tilde{C} \\
j_{t}= & \tilde{B} y_{t}+\left(\bar{\Lambda}+\left(R+r^{f}\right) C\right) x_{t-1}+A_{x y}\left((I-\Pi) y_{t}-\tilde{C} x_{t-1}\right)+ \\
& \tilde{C} A_{y y}\left((I-\Pi) y_{t}-\tilde{C} x_{t-1}\right) \\
\equiv & S_{x} x_{t-1}+S_{y} y_{t} \\
d_{t}= & -\frac{1}{2} x_{t-1} \bar{\Lambda} x_{t-1}-\frac{1}{2} \bar{\rho}^{-1} x_{t-1}^{\top} C x_{t-1}+ \\
& \frac{1}{2}\left((I-\Pi) y_{t}-\tilde{C} x_{t-1}\right)^{\top} A_{y y}\left((I-\Pi) y_{t}-\tilde{C} x_{t-1}\right) .
\end{aligned}
$$

Here,

$$
\begin{aligned}
& S_{x}=\bar{\Lambda}+\left(R+r^{f}\right) C-A_{x y} \tilde{C}-\tilde{C}^{\top} A_{y y} \tilde{C} \\
& S_{y}=\tilde{B}+A_{x y}(I-\Pi)+\tilde{C}^{\top} A_{y y}(I-\Pi) .
\end{aligned}
$$

The value of $x$ attaining the maximum is given by

$$
x_{t}=J^{-1} j_{t}
$$


and the maximal value is

$$
\begin{aligned}
\frac{1}{2} j_{t} J^{-1} j_{t}+d_{t} & =V\left(x_{t-1}, y_{t}\right)-A_{0} \\
& =-\frac{1}{2} x_{t-1}^{\top} A_{x x} x_{t-1}+x_{t-1}^{\top} A_{x y} y_{t}+\frac{1}{2} y_{t}^{\top} A_{y y} y_{t}
\end{aligned}
$$

The unknown matrices have to satisfy a system of equations encoding the equality of all coefficients in (A.51). Thus,

$$
\begin{aligned}
-\bar{\rho}^{-1} A_{x x} & =S_{x}^{\top} J^{-1} S_{x}-\bar{\Lambda}-\bar{\rho}^{-1} C+\tilde{C}^{\top} A_{y y} \tilde{C} \\
\bar{\rho}^{-1} A_{x y} & =S_{x}^{\top} J^{-1} S_{y}-\tilde{C}^{\top} A_{y y}(I-\Pi) \\
\bar{\rho}^{-1} A_{y y} & =S_{y}^{\top} J^{-1} S_{y}+(I-\Pi)^{\top} A_{y y}(I-\Pi) .
\end{aligned}
$$

For our purposes, the more interesting observation is that the optimal position $x_{t}$ is rewritten as

$$
x_{t}=x_{t-1}+\underbrace{\left(I-J^{-1} S_{x}\right)}_{M^{\text {rate }}}(\underbrace{\left(I-J^{-1} S_{x}\right)^{-1}\left(J^{-1} S_{y}\right) y_{t}}_{\text {aim }_{t}=M^{\text {aim }} y_{t}}-x_{t-1}) \text {. }
$$




\section{References}

Acharya, Viral, and Lasse Heje Pedersen, 2005, Asset pricing with liquidity risk, Journal of Financial Economics 77, 375-410.

Almgren, Robert, and Neil Chriss, 2000, Optimal execution of portfolio transactions, Journal of Risk 3, 5-39.

Amihud, Yakov, and Haim Mendelson, 1986, Asset pricing and the bid-ask spread, Journal of Financial Economics 17, 223-249.

Balduzzi, P., and A.W. Lynch, 1999, Transaction costs and predictability: some utility cost calculations, Journal of Financial Economics 52, 47-78.

Bertsimas, Dimitris, and Andrew W. Lo, 1998, Optimal control of execution costs, Journal of Financial Markets 1, 1-50.

Breen, William .J., Laurie S. Hodrick, and Robert A. Korajczyk, 2002, Predicting Equity Liquidity, Management Science 48, 470-483.

Campbell, John Y., and Luis M. Viceira, 2002, Strategic Asset Allocation Portfolio Choice for Long-Term Investors. (Oxford University Press, ).

Constantinides, George M., 1986, Capital market equilibrium with transaction costs, Journal of Political Economy 94, 842-862.

Engle, Robert, and Robert Ferstenberg, 2007, Execution risk, Journal of Portfolio Management 33, 34-45.

Engle, Robert, Robert Ferstenberg, and Jeffrey Russell, 2008, Measuring and Modeling Execution Cost and Risk, Working paper, University of Chicago.

Gârleanu, Nicolae, 2009, Portfolio choice and pricing in imperfect markets, Journal of Economic Theory 144, 532-564. 
Gârleanu, Nicolae, Lasse Heje Pedersen, and Allen Poteshman, 2009, Demand-based option pricing, Review of Financial Studies 22, 4259-4299.

Greenwood, Robin, 2005, Short and long term demand curves for stocks: Theory and evidence, Journal of Financial Economics 75, 607-650.

Grinold, Richard, 2006, A dynamic model of portfolio management, Journal of Investment Management 4, 5-22.

Grossman, Sanford, and Merton Miller, 1988, Liquidity and market structure, Journal of Finance 43, 617-633.

Heaton, John, and Deborah Lucas, 1996, Evaluating the effects of incomplete markets on risk sharing and asset pricing, Journal of Political Economy 104, 443-487.

Jang, B.G., H.K. Koo, H. Liu, and M. Loewenstein, 2007, Liquidity premia and transaction costs, Journal of Finance 62, 2329-2366.

Lillo, Fabrizio, J. Doyne Farmer, and Rosario N. Mantegna, 2003, Master Curve for PriceImpact Function, Nature 421, 129-130.

Liu, H., 2004, Optimal consumption and investment with transaction costs and multiple assets, Journal of Finance 59, 289-338.

Ljungqvist, Lars, and Thomas Sargent, 2004, Recursive Macroeconomic Theory, 2nd edition. (MIT press, ).

Lo, Andrew, H. Mamaysky, and J. Wang, 2004, Asset prices and trading volume under fixed transaction costs, Journal of Political Economy 112, 1054-1090.

Lynch, A.W., and P. Balduzzi, 2000, Predictability and transaction costs: the impact on rebalancing rules and behavior, Journal of Finance pp. 2285-2309. 
Lynch, Anthony, and Sinan Tan, 2011, Explaining the magnitude of liquidity premia: The roles of return predictability, wealth shocks, and state-dependent transaction costs, Journal of Finance 66, 1329-1368.

Markowitz, Harry M., 1952, Portfolio selection, The Journal of Finance 7, 77-91.

Obizhaeva, Anna, and Jiang Wang, 2006, Optimal trading strategy and supply/demand dynamics, Working paper, MIT.

Perold, Andre, 1988, The implementation shortfall: Paper versus reality, Journal of Portfolio Management 14, 4-9.

Vayanos, Dimitri, 1998, Transaction costs and asset prices: A dynamic equilibrium model, Review of Financial Studies 11, 1-58.

Vayanos, Dimitri, and Jean-Luc Vila, 1999, Equilibrium interest rate and liquidity premium with transaction costs, Economic Theory 13, 509-539. 


\begin{tabular}{lrrrr} 
Commodity & $\begin{array}{c}\text { Average Price } \\
\text { Per Contract }\end{array}$ & $\begin{array}{c}\text { Standard } \\
\text { Deviation of } \\
\text { Price Changes }\end{array}$ & $\begin{array}{c}\text { Contract } \\
\text { Multiplier }\end{array}$ & $\begin{array}{c}\text { Daily Trading } \\
\text { Volume } \\
\text { (Contracts) }\end{array}$ \\
\hline Aluminum & 44,561 & 637 & 25 & 9,160 \\
Cocoa & 15,212 & 313 & 10 & 5,320 \\
Coffee & 38,600 & 1,119 & 37,500 & 5,640 \\
Copper & 80,131 & 2,023 & 25 & 12,300 \\
Crude & 40,490 & 1,103 & 1,000 & 151,160 \\
Gasoil & 34,963 & 852 & 100 & 37,260 \\
Gold & 43,146 & 621 & 100 & 98,700 \\
Lead & 23,381 & 748 & 25 & 2,520 \\
Natgas & 50,662 & 1,932 & 10,000 & 46,120 \\
Nickel & 76,530 & 2,525 & 6 & 1,940 \\
Silver & 36,291 & 893 & 5,000 & 43,780 \\
Sugar & 10,494 & 208 & 112,000 & 25,700 \\
Tin & 38,259 & 903 & 5 & NaN \\
Unleaded & 47,967 & 1,340 & 42,000 & 11,320 \\
Zinc & 36,513 & 964 & 25 & 6,200 \\
& & & & \\
\hline
\end{tabular}

Table I: Summary Statistics. For each commodity used in our empirical study, the first column reports the average price per contract in U.S. dollars over our sample period 01/01/1996-01/23/2009. For instance, since the average gold price is $\$ 431.46$ per ounce, the average price per contract is $\$ 43,146$ since each contract is for 100 ounces. Each contract's multiplier (100 in the case of gold) is reported in the third column. The second column reports the standard deviation of price changes. The fourth column reports the average daily trading volume per contract, estimated as the average daily volume of the most liquid contract traded electronically and outright (i.e., not including calendar-spread trades) in December 2010. 


\begin{tabular}{|c|c|c|c|c|}
\hline & \multicolumn{2}{|c|}{$\begin{array}{c}\text { Panel A: } \\
\text { Benchmark } \\
\text { Transaction Costs }\end{array}$} & \multicolumn{2}{|c|}{$\begin{array}{c}\text { Panel B: } \\
\text { High Transaction } \\
\text { Costs }\end{array}$} \\
\hline & Gross SR & Net SR & Gross SR & Net SR \\
\hline Markowitz & 0.83 & -9.84 & 0.83 & -10.11 \\
\hline Dynamic optimization & 0.62 & 0.58 & 0.58 & 0.53 \\
\hline \multicolumn{5}{|l|}{ Static optimization } \\
\hline Weight on Markowitz $=10 \%$ & 0.63 & -0.41 & 0.63 & -1.45 \\
\hline Weight on Markowitz = 9\% & 0.62 & -0.24 & 0.62 & -1.10 \\
\hline Weight on Markowitz = 8\% & 0.62 & -0.08 & 0.62 & -0.78 \\
\hline Weight on Markowitz = 7\% & 0.62 & 0.07 & 0.62 & -0.49 \\
\hline Weight on Markowitz $=6 \%$ & 0.62 & 0.20 & 0.62 & -0.22 \\
\hline Weight on Markowitz = 5\% & 0.61 & 0.31 & 0.61 & 0.00 \\
\hline Weight on Markowitz = 4\% & 0.60 & 0.40 & 0.60 & 0.19 \\
\hline Weight on Markowitz = 3\% & 0.58 & 0.46 & 0.58 & 0.33 \\
\hline Weight on Markowitz = 2\% & 0.52 & 0.46 & 0.52 & 0.39 \\
\hline Weight on Markowitz = 1\% & 0.36 & 0.33 & 0.36 & 0.31 \\
\hline
\end{tabular}

Table II: Performance of Trading Strategies Before and After Transaction Costs. This table shows the annualized Sharpe ratio gross and net of trading costs for the optimal trading strategy in the absence of trading costs ("no TC"), our optimal dynamic strategy ("optimal"), and a strategy that optimizes a static one-period problem with trading costs ("static"). Panel A illustrates this for a low transaction cost parameter, while Panel B has a high one. 


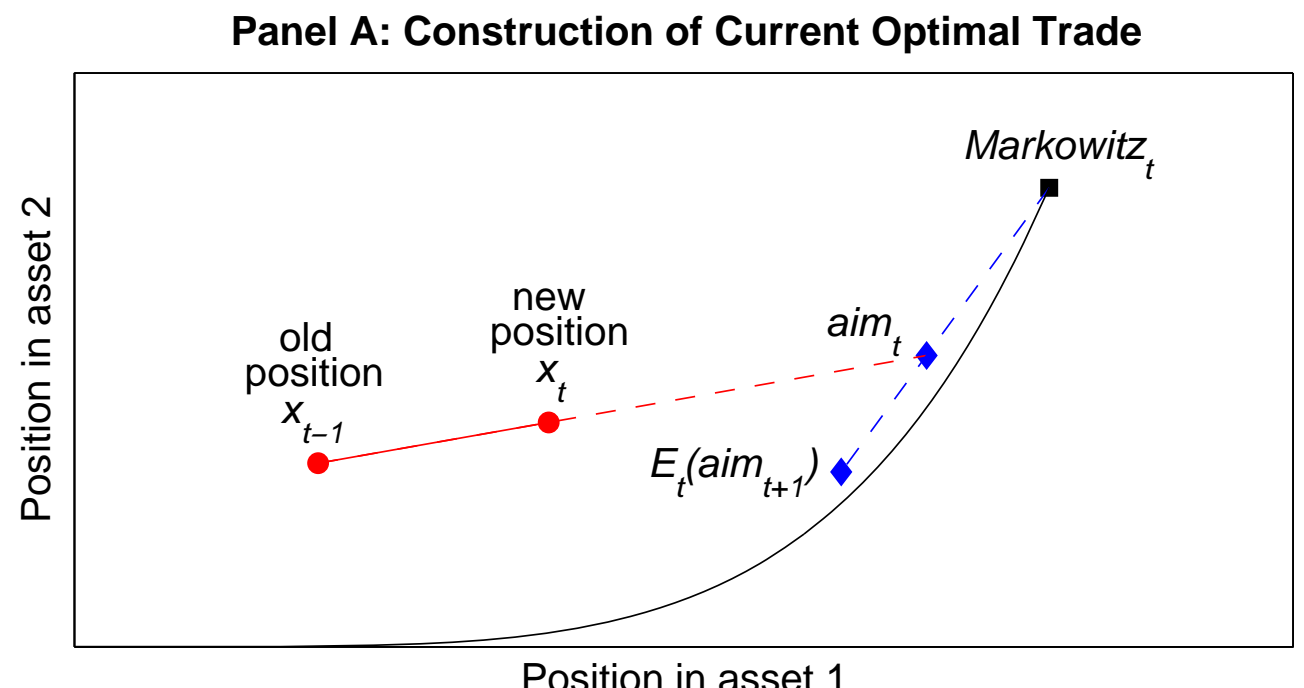

Figure 1: Optimal Trading Strategy: Aim in Front of the Target. This figure shows how the optimal trade moves the portfolio from the existing position $x_{t-1}$ towards the aim portfolio, trading only part of the way to the aim portfolio to limit transactions costs. The aim portfolio is an average of the current Markowitz portfolio (the optimal portfolio in the absence of transaction costs) and the expected future aim portfolio, which reflects how the Markowitz portfolio is expected to mean-revert to its long-term level (the lower, left corner of the figure). 

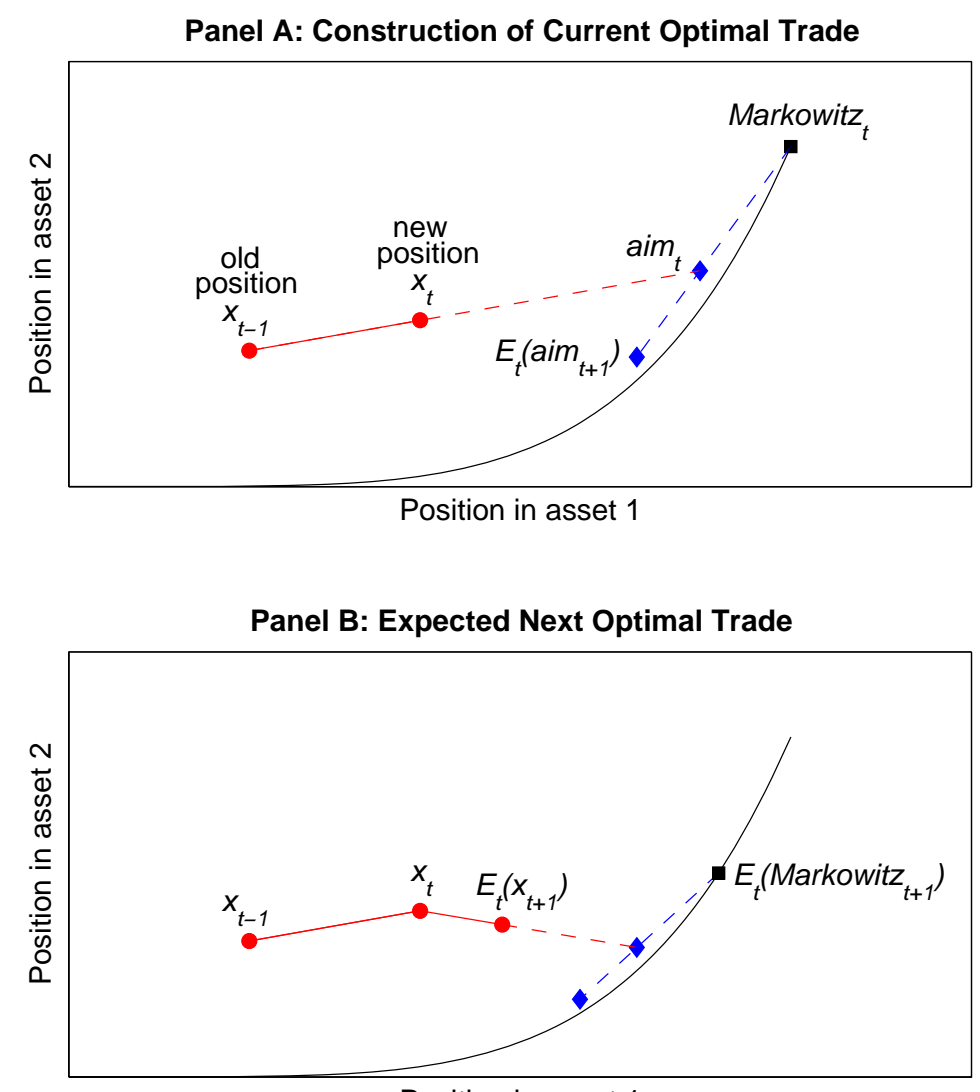

Position in asset 1

Panel C: Expected Evolution of Portfolio

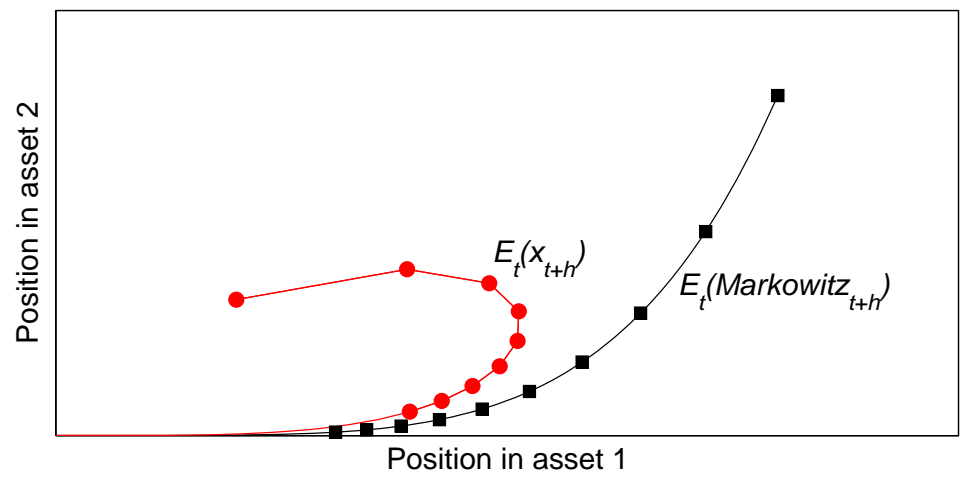

Figure 2: Aim in Front of the Target by Underweighting Fast-Decay Factors. This figure shows how the optimal trade moves from the existing position $x_{t-1}$ towards the aim, which puts relatively more weight on assets loading on persistent factors. Relative to the Markowitz portoflio, the weight in the aim of asset 2 is lower because asset 2 has a fasterdecaying alpha, as is apparent in the lower expected weight it receives in future Markowitz portfolios. 


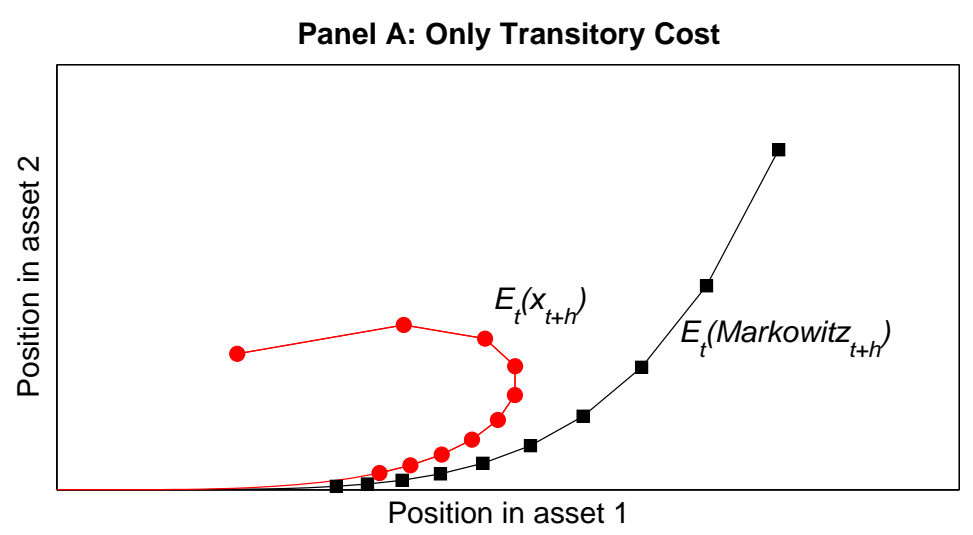

Panel B: Persistent and Transitory Cost
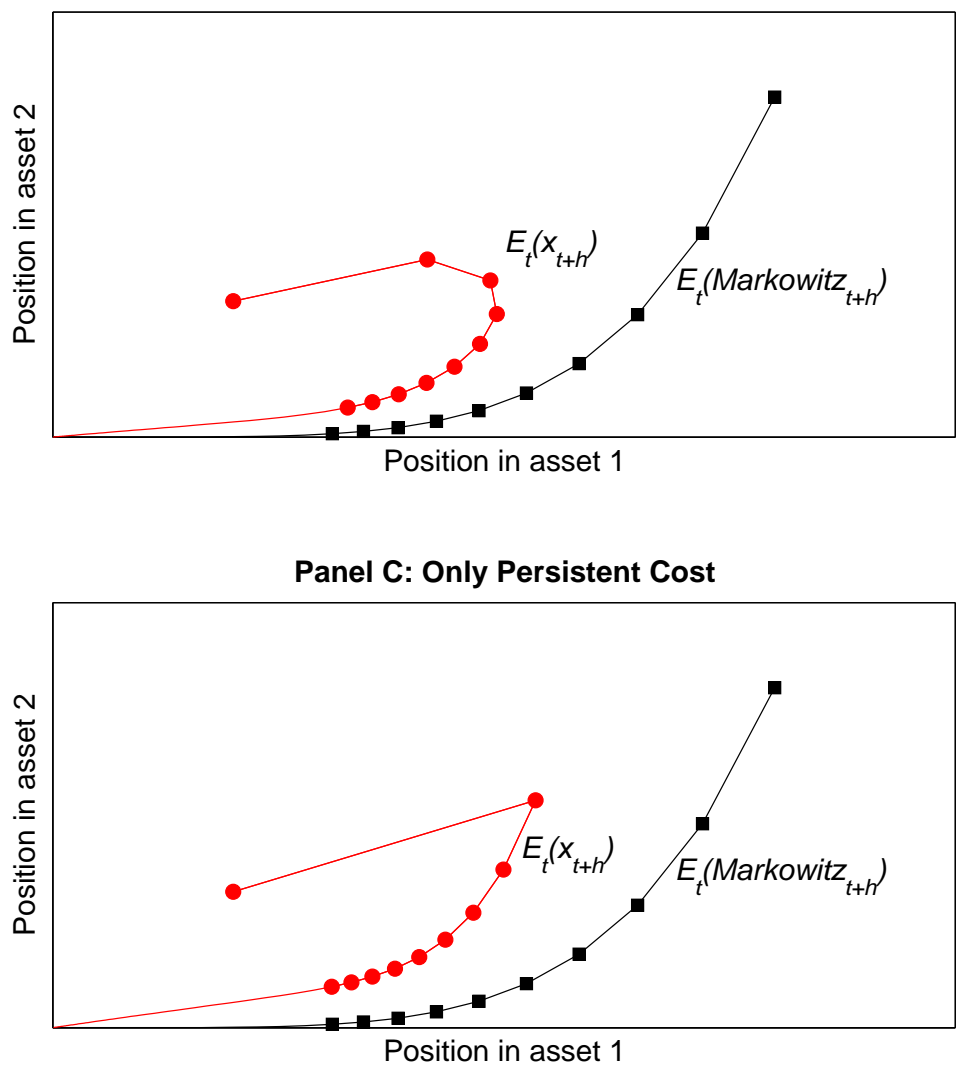

Figure 3: Aim in Front of the Target with Persistent Costs. This figure shows the optimal trade when part of the transaction cost is persistent. In panel A, the entire cost is transitory, as in Figures 1 and 2. In panel B, half of the cost is transitory, while the other half is persistent, with a half life of 6.9 periods. In panel $\mathrm{C}$, the entire cost is persistent. 

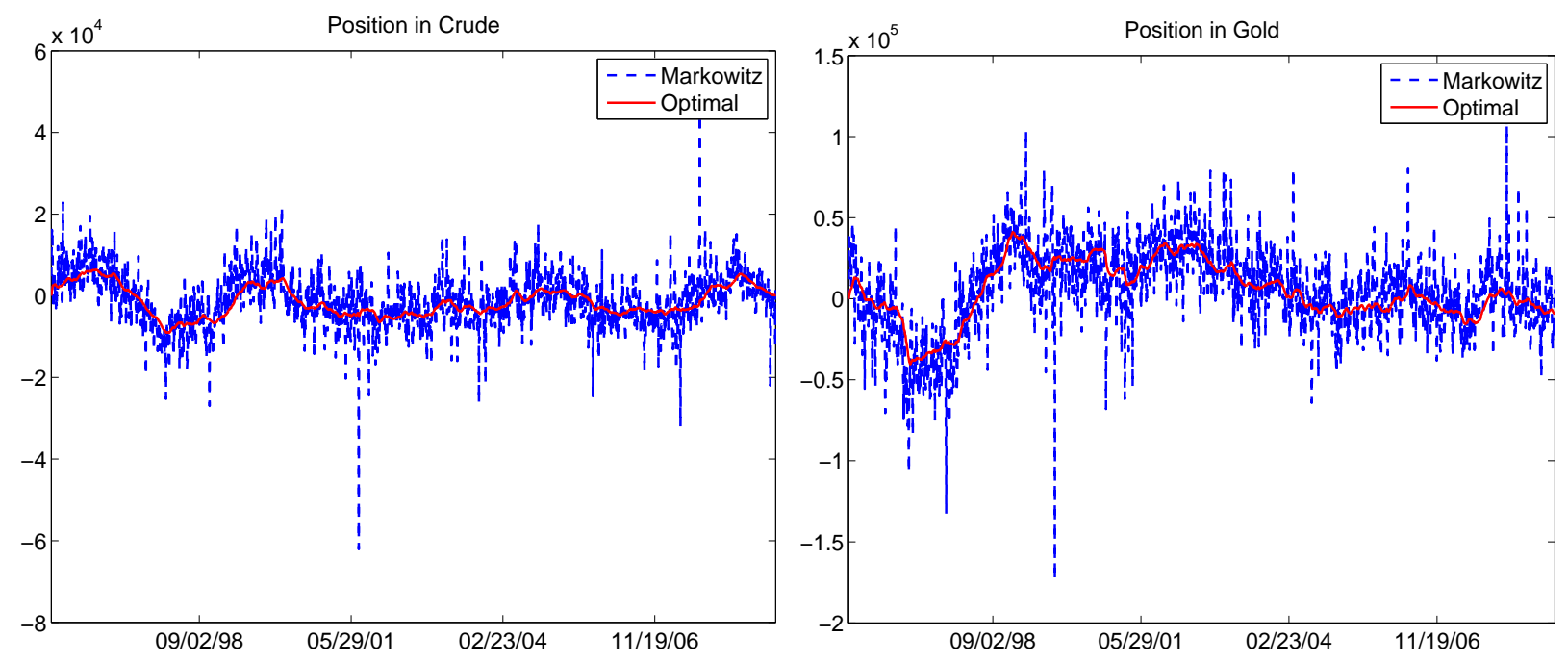

Figure 4: Positions in Crude and Gold Futures. This figure shows the positions in crude and gold for the the optimal trading strategy in the absence of trading costs ("Markowitz") and our optimal dynamic strategy ("optimal"). 


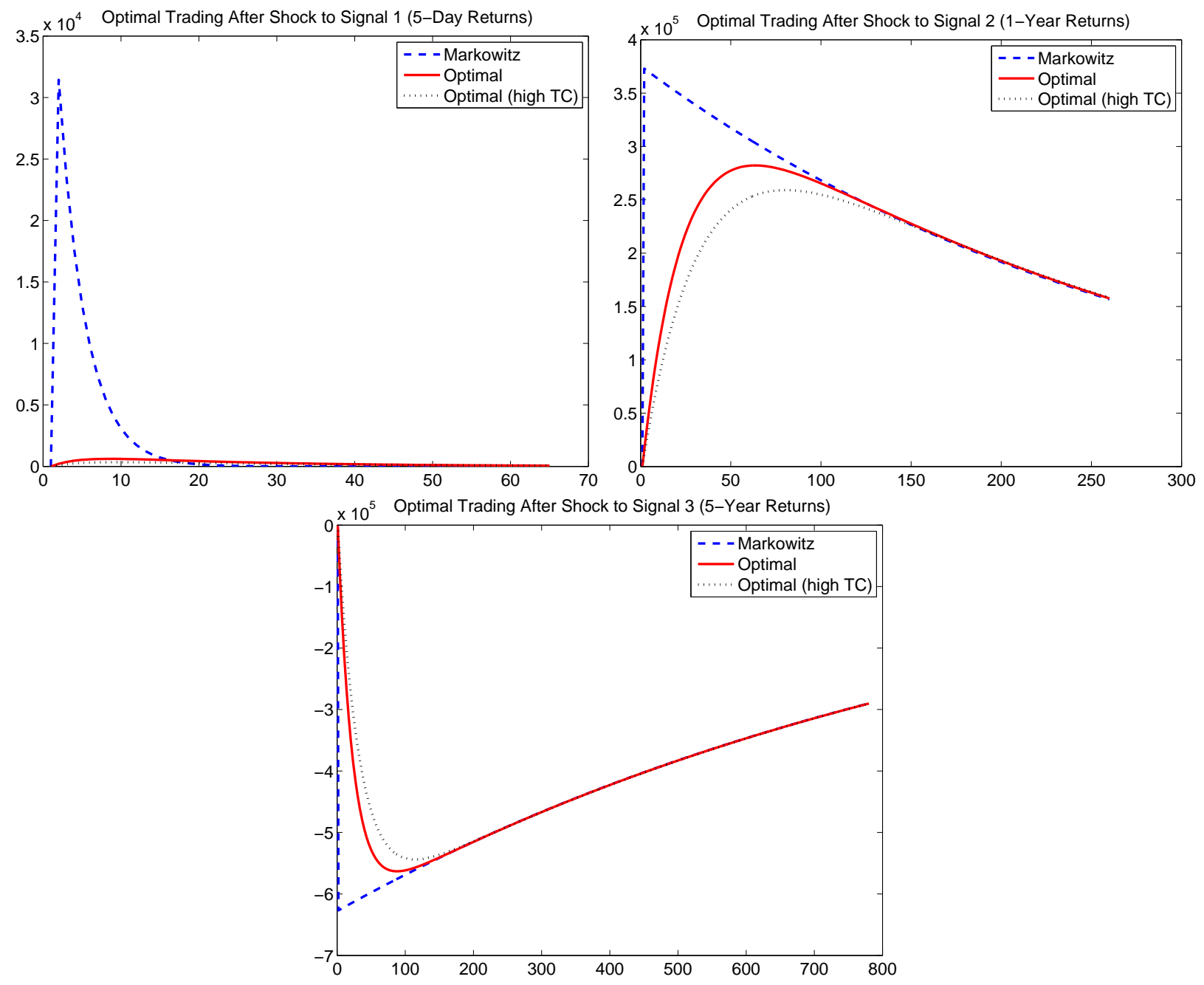

Figure 5: Optimal Trading in Response to Shock to Return Predicting Signals. This figure shows the response in the optimal position following a shock to a return predictor as a function of the number of days since the shock. The top left panel does this for a shock to the fast five-day return predictor, the top right panel considers a shock to the 12-month return predictor, and the bottom panel to the five-year predictor. In each case, we consider the response of the optimal trading strategy in the absence of trading costs ("Markowitz") and our optimal dynamic strategy ("optimal") using high and low transactions costs. 